\title{
Information acquisition strategies and the cognitive structure of arithmetic
}

\author{
LISA M. STEVENSON and RICHARD A. CARLSON \\ Pennsylvania State University, University Park, Pennsylvania
}

\begin{abstract}
In three experiments, we examined the sensitivity of information acquisition strategies to the underlying cognitive structure of arithmetic tasks. Previous work has shown that individuals solve arithmetic problems more quickly when they consider operators before operands. The operators establish the goal, and the operands are then assimilated into that goal. In the present experiments, participants performed arithmetic tasks in which they controlled the display of operators and operands, using separate keystrokes. The participants chose to see the operator before the operands in most conditions. This ordering was more likely when task constraints made it easier to achieve, when feedback emphasized execution time, and in a more complex multiple-step task. These results extend previous research on strategy selection and information acquisition, supporting the idea that coordination between the environment and ongoing cognition is an important feature of cognitive skill.
\end{abstract}

In some cognitive tasks, coordinating acquisition of information from the environment with ongoing mental processes is relatively straightforward. In these cases, the needed information is continually available in the environment, and the timing of skilled information acquisition activities can be triggered by ongoing mental processes. Reading is one example. The information needed is continually available, and it is typically organized in a well-learned, conventional manner. The mental processes tracking the reading can, therefore, trigger the perceptual and attentional processes needed to pick up the next relevant word, and skilled readers accomplish this coordination with little effort or deliberation (e.g., Just \& Carpenter, 1980). However, there are many tasks in which people must take some deliberate action to make necessary information available. While working at a computer, for example, one might have to click a mouse button to display some piece of information. In other cases, visual search might be necessary to find the relevant information. In the present research, we examined how people are able to strategically coordinate their information acquisition activities with ongoing mental processes in the face of cognitive and environmental constraints.

The different orders in which individuals pick up information to perform a task are likely to vary in their efficiency. For example, Bovair and Kieras (1991) reviewed evidence that individuals follow procedural instructions more effectively when elements are presented in the order in which they must be used to perform the proce-

The experiments reported in this article were based on the Master's thesis of the first author. Portions of this work were presented at the Eastern Psychological Association annual meeting, Boston, March 8, 2002. Correspondence concerning this article should be addressed to L. M. Stevenson, 604 Moore Building, University Park, PA 16802 (e-mail:1ms152@psu.edu). dure. They noted that although some evidence supports this principle at the level of elements required for single procedural steps, interpreting previous research on this issue is difficult, because the optimal order of elements is not always obvious and because there may be conflicts between procedural and grammatical orderings for instructions. In the present experiments, we addressed this issue by making use of a paradigm in which there is previous evidence concerning the optimal ordering of procedural elements and in which concerns about textual and grammatical constraints are minimal.

Our paradigm is based on previous research that suggests that there is an optimal order in which information should be picked up in arithmetic tasks, such as adding a pair of digits. In this task, one could consider the operator (addition) first, consider the operands (digits) first, or consider these elements in the order operand-operatoroperand. Sohn and Carlson (1998) presented the participants with single-step Boolean and arithmetic tasks in which the elements appeared in each of these orders. For both problem types, the participants were fastest (measured from the point at which all information was available) when they saw the operator first. Carlson and Sohn (2000) demonstrated a similar effect in multiple-step problems in both arithmetic and spatial domains. In these problems, the result of one step served as an operand for the subsequent step. In both the arithmetic and the spatial tasks, participants performed most fluently when the operator appeared before the new operand at each step. This result was found when both operator and operand were acquired on line and when one or the other was already in working memory. As long as the operators were considered first, performance was more fluent.

Carlson and Sohn (2000; Sohn \& Carlson, 1998) interpreted their results in terms of the procedural frame hypothesis. This hypothesis suggests that instantiating a 
goal establishes a frame into which operands may be assimilated and contrasts with the view that operators and operands serve similar roles as retrieval cues for arithmetic facts (e.g., Reder \& Schunn, 1996). In arithmetic, this means that participants must intend to perform an operation before they can effectively consider the operands upon which that operation is to be performed.

These findings are consistent with other research in the task-switching and number theory literatures. In task switching, knowledge of and the opportunity to prepare for the upcoming task generally decreases response time (RT; e.g., Rogers \& Monsell, 1995; Sohn \& Carlson, 2000). Similarly, knowledge of the upcoming operator to be used allows for faster solution times. This may be because it allows for the appropriate encoding of the numbers. Some researchers have argued that numbers must be coded in a manner specific to the task for which the numbers will be used (e.g., Clark \& Campbell, 1991; Dehaene, 1992). In Dehaene's triple-code model, for example, comparison and addition require that numbers be coded in two different ways; comparison tasks require an analogue magnitude representation, whereas simple addition is more likely to require an auditory verbal word frame. If the operands are seen first, participants may begin to encode the numbers by using one representation, later finding that they need to recode those numbers.

This previous research has shown that fluent performance of cognitive tasks depends on appropriately ordering the acquisition of required information. Failure to achieve this ordering may reduce fluency in at least two ways. First, inappropriate ordering introduces a working memory load, adding component processes to the task. When information is picked up as it is needed, individuals can use it immediately, coding operands in a manner appropriate for the task. When it is picked up too early, however, participants must remember the information until it is time to use it. Encoding information in a format suitable for storing it in working memory, managing rehearsal, and retrieving the information when it is needed may slow processing, as may any slack time in which information is simply stored. Second, these working memory processes may hurt performance not just by adding component processes, but also because of conflicts between working memory management and taskcritical processes, such as instantiating a goal to apply the appropriate operator.

In previous research, the experimenter has determined the sequence in which problem elements become available. Therefore, it is not clear whether or under what conditions participants would be able to optimally arrange their information acquisition activities when left to their own devices. In the present study, we addressed this question by allowing participants to request the display of operators and operands in arithmetic problems. If information acquisition strategies are sensitive to the cognitive constraints suggested by the procedural frame hypothesis, individuals should choose to display operators in advance of operands.

\section{Constraints on Information Acquisition Strategies}

The structure of cognitive processes may constrain information acquisition strategies at several time scales. On the scale of tens of seconds, the goal structure adopted for a task specifies the sequence in which subgoals will be completed and, thus, the order in which information must be considered. In tasks of even moderate complexity, working memory capacity can easily be exceeded if information acquisition is not coordinated with this order. Indeed, the order in which individuals choose to acquire information is often used as an indicator of underlying cognitive strategy in problem-solving and decision tasks (e.g., Cary \& Carlson, 1999; Payne, 1976). Even when working memory capacity is not exceeded, individuals may prefer information acquisition strategies that minimize the need to store information. Ballard, Hayhoe, Pook, and Rao (1997) reviewed evidence that individuals adopt such minimal memory strategies for sequential tasks, picking up information just as it is needed, rather than storing it in working memory. At a scale of a few seconds, the demands of performing one task may constrain the time at which an individual can effectively consider a stimulus for a subsequent task. For example, Pashler and Johnston (1989) demonstrated that some combinations of tasks performed in quick succession resulted in high levels of interference when stimuli appeared close together in time but in much better performance as the time between stimuli increased. The present experiments are concerned with a still finer grained level of analysis, examining the time course with which elements of a single-step task (i.e., a task that might be represented in terms of a single goal or production) are considered.

The order in which participants acquire information may also reflect interactions between cognitive structures and environmental constraints. For example, Cary and Carlson (1999) examined the strategic ordering of information acquisition in a complex, multiple-step arithmetic task. Their procedure required that participants explicitly select information from several screen displays that appeared one at a time at the participant's request. They found that the order in which the participants picked up information depended in part on the presence or absence of an external aid (paper and pencil). The participants who had paper and pencil requested information in sequences that matched the conceptual structure of problems, whereas those without that memory aid used sequences that required less cognitive effort (e.g., strategies that required holding less information in working memory). These findings demonstrate that cognitive structures-here, problem-solving strategies-may be adapted to constraints on the perceptual availability of information.

Although it might seem obvious that individuals coordinate cognitive structures and information pickup strategies to achieve effective performance, there are several reasons for questioning whether this generality 
holds at the fine-grained level of individual goals. First, the selection of execution strategies for simple tasks, such as arithmetic, appears to be implicit rather than deliberate (Reder \& Schunn, 1996; Siegler, Adolph, \& Lemaire, 1996). Second, it is not clear that individuals have metacognitive access to the fine-grained structure of mental processes that perform simple tasks such as mental arithmetic (although recent research by Shin \& Rosenbaum, 2002, suggests that the duration of such processes can be adjusted). Third, under some circumstances, the representation needed to guide an optimal information acquisition strategy may conflict with the representation needed to optimally perform the task. For example, the experiments here include conditions in which optimal performance requires considering arithmetic operators in advance of operands, but the optimal information acquisition strategy involves requesting operands before requesting operators. This is analogous to baking a dessert that takes an hour and a main course that takes $15 \mathrm{~min}$. Although one will need the main course first, starting the dessert (like requesting the operands) before the main course (like requesting the operator) will allow the food to be ready in the order in which it is needed, minimizing the time between the availability of the two dishes. However, these two representations of the meal (dessert-main-course, main-course-dessert) conflict.

\section{Present Experiments}

In the present experiments, the primary manipulation was a delay between requests and presentations of operators and operands. This manipulation requires that participants adjust the timing of their requests to maintain optimal sequencing of the information that they need in order to complete each problem. In each of the present experiments, the participants requested the operators and the operands for arithmetic problems by pressing two separate keys. The delay between request and display was either short $(200 \mathrm{msec})$ or long $(1,500 \mathrm{msec})$, and each of the four possible assignments of delays to operator and operand requests was used in $2 \times 2$ betweensubjects designs. A between-subjects design was used to provide the clearest data on strategy selection, given the timing delays. This design allowed the participants to develop with practice the optimal strategy for their given delay conditions.

On the basis of the procedural frame hypothesis, the optimal ordering of information would be to see the operator before the operands. The participants in all the conditions could accomplish this by requesting the operator, waiting until it appeared, and then requesting the operands. The participants might also be expected to organize their requests so that the operator would precede the operands by only a few hundred milliseconds, juxtaposing activation of the procedure with the availability of operands (Carlson, 1997). Thus, when a short delay follows the operator request but a long delay follows the operand request, the participants should request the operands first but should request the operator soon enough that it would be seen first.

Between experiments, we also varied the feedback received by the participants. In Experiment 1, latency feedback reflected the entire time taken to complete each problem, from the first request until the answer. In Experiment 2 , feedback reflected only the time between the display of the final piece of information (operator or operands) and the answer. This measure of latency thus includes only the time required to encode the last element and to execute whatever process realizes the calculation. These alternative feedback procedures may encourage individuals to optimize different portions of the task and, therefore, lead people to different strategies for timing information requests. Finally, we investigated both single- and multiple-step problems. Multiple-step problems are cognitively more demanding (e.g., requiring greater use of working memory) and may, thus, place a greater premium on optimal timing and coordination.

\section{EXPERIMENT 1}

In Experiment 1, we asked whether participants who control the order of the presentation of information would choose to see the operator first, thus creating the opportunity for optimal coordination between environmental information and ongoing mental processes. Furthermore, we explored how participants would adjust to the delays between the requests for and the displays of information. It was hypothesized that the participants would choose to see the operator before the operands, perhaps organizing their responses so that the operator and the operands would appear close together temporally. Together, these hypotheses suggested that the participants in the short operator delay/long operand delay condition would request the operands first but would request the operator soon enough that it would be seen first.

\section{Method}

Forty-nine undergraduate psychology students participated in exchange for a small amount of course credit. Arithmetic problems were presented by PC-compatible computers that used VGA color monitors and were programmed with the Micro Experimental Lab system (Schneider, 1988). One participant more than necessary was recruited, resulting in 13 participants in one of the four conditions.

Each trial was preceded by the presentation of parentheses in the middle of the screen. After the appropriate request, the operands appeared inside the parentheses. The operator appeared either to the right or to the left of the parentheses, counterbalanced across participants.

The first block of problems served both to introduce the participants to the task and to provide a check on random assignment. The participants pressed the space bar to request the necessary information. After a 200-msec delay, both the operator and the operands appeared at the same time on the screen. They were visible for $400 \mathrm{msec}$, after which they were masked.

For the remaining blocks, the participants requested the operator and the operands separately. The "f $\mathrm{f}$ " and " $\mathrm{j}$ " keys on the computer keyboard were labeled as "O" for operator and "\#" for operands. The mapping of these keys to the display was such that the key used 
to request a piece of information was on the same side as the displayed information.

The participants were randomly assigned to one of four conditions, created by crossing the delay following the operator request (either 200 or $1,500 \mathrm{msec}$ ) with the delay following the operand request (either 200 or $1,500 \mathrm{msec}$ ). Following the first block of problems, the participants were told that there might be delays following the operator and the operand requests. Although they were not told the duration of the delay, they were told that the delays would remain consistent throughout the remainder of the experiment.

Each piece of information was displayed for $200 \mathrm{msec}$ and then masked. This was intended to make the timing of the acquisition of information critical and to discourage the participants from viewing both pieces of information at the same time. The participants were instructed to solve each problem as quickly as possible while still maintaining a high level of accuracy, and they received accuracy feedback for each trial and RT feedback for correct trials. RT feedback was given as the total time, in seconds, from the first request for information to the time the answer was entered with the computer keypad.

Each participant solved a total of 10 blocks of 80 problems each, including the practice block. Four operations were used: sum, difference, minimum, and maximum. These were displayed on the screen as "sum," "dif," "min," and "max." For "sum" problems, the participants simply added the two operands. For "dif" problems, the participants found the absolute difference between the two operands. For "min" and "max" problems, the participants compared the two operands and were instructed to answer with either the smaller (minimum) or the larger (maximum). Each operation was shown 20 times per block in a random order. Seeing the same operation twice in a row was, therefore, possible. Operands were selected randomly from the set of one to nine, with the stipulation that the correct response to each problem was required to be a single-digit number. If the randomly selected operands did result in a correct response that was greater than nine, new operands were randomly generated until that condition was met. The task was self-paced, and each problem was initiated by the first request of the participant. The intertrial interval was, therefore, determined by the participant.

\section{Results and Discussion}

The participants chose to view information in the optimal order as suggested by the procedural frame hypothesis, operator before operands, the majority of the time (66\% of the problems), although this varied as a function of experimental condition. The participants in the short operator delay/short operand delay condition saw the operator first $91 \%$ of the time; the participants in the short operator delay/long operand delay condition saw the operator first $99 \%$ of the time. The participants in the long operator delay/long operand delay condition $(M=$ $60 \%$ ) were less likely to see the operator first than were these two groups; the participants in the long operator delay/short operand delay condition rarely saw the oper- ator first $(M=13 \%)$. In an operator delay $\times$ operand delay $\times$ block mixed-factorial analysis of variance (ANOVA), this operator $X$ operand delay interaction was shown to be significant $\left[F(1,45)=4.05, M S_{\mathrm{e}}=4.05, p<.01\right.$; partial $\eta^{2}=.03$ ], subsuming main effects of operator delay $\left[F(1,45)=103.56, M S_{\mathrm{e}}=37.03, p<.001 ;\right.$ partial $\left.\eta^{2}=.7\right]$ and operand delay $\left[F(1,45)=8.56, M S_{\mathrm{e}}=8.56\right.$, $p<.001$; partial $\eta^{2}=.35$ ]. A block $\times$ operator delay interaction was also found to be significant $[F(8,360)=$ 2.26, $M S_{\mathrm{e}}=0.07, p<.05$; partial $\left.\eta^{2}=.05\right]$. Analyses of the simple main effects revealed that the participants in the short operator delay conditions became more likely to see the operator first with practice, whereas the participants in the long operator delay conditions did not show a practice effect.

For the equal operator and operand delay conditions, request and display orders had to match, although that did not need be true in the two mixed-delay conditions. The interesting case is the short operator delay/long operand delay condition, in which the participants could request the operands first but still see the operator first. The participants in this condition requested the operator first on only $56 \%$ of the trials, although they saw the operator first on $99 \%$ of the trials. This means that on $43 \%$ of the trials, the participants requested the operands first but then requested and saw the operator during the operand delay. The request and display orders for all the conditions are shown in Table 1.

The participants tended to adopt consistent display orders. By the end of practice (the last two blocks of problems), a total of 42 participants had seen either the operator or the operands first on at least $85 \%$ of the trials. Thirty of these participants consistently saw the operator first, whereas only 12 consistently saw the operands first. This was found to differ significantly from chance $\left[\chi^{2}(1, N=42)=7.71, p<.01\right]$.

To achieve these display orders, the majority of these participants established a consistent request order by the last two blocks of trials. All but 9 participants requested either the operator or the operands first on more than $85 \%$ of the trials. For those 9 participants, the mean interrequest interval was $33 \mathrm{msec}$ for the last two blocks, as compared with $262 \mathrm{msec}$ for the other 40 participants. Thus, these participants' requests were essentially simultaneous. Of the 40 participants who consistently requested either the operator or the operands first, 32 consistently requested the operator first, and 8 consistently requested the operands first $\left[\chi^{2}(1, N=40)=14.4, p<.001\right]$.

Table 1

Proportion of Operators Displayed and Requested First as a Function of Delay Condition

\begin{tabular}{|c|c|c|c|c|c|c|c|c|}
\hline \multirow[b]{3}{*}{ Experiment } & \multicolumn{8}{|c|}{ Delay Condition (Operator/Operand) } \\
\hline & \multicolumn{2}{|c|}{ Short/Short } & \multicolumn{2}{|c|}{ Short/Long } & \multicolumn{2}{|c|}{ Long/Short } & \multicolumn{2}{|c|}{ Long/Long } \\
\hline & Display & Request & Display & Request & Display & Request & Display & Request \\
\hline 1 & .91 & .91 & .99 & .56 & .13 & .76 & .60 & .60 \\
\hline 2 & .79 & .79 & .97 & .53 & .58 & .74 & .86 & .86 \\
\hline 3 & .83 & .83 & .82 & .66 & .60 & .78 & .59 & .59 \\
\hline
\end{tabular}


Accuracy does not account for any differences in the ordering of information displays among the groups. Accuracy was high both before and after the introduction of the experimental manipulation. For the first block of trials, accuracy was high $(M=95 \%)$, and it did not vary as a function of operator delay $[F(1,45)<1]$ or operand delay $(p>.2)$ in an operator delay $\times$ operand delay between-subjects ANOVA. The operator $\times$ operand delay interaction did not near significance $[F(1,45)<1]$. After the introduction of the experimental manipulation, accuracy remained high $(M=93 \%)$, and it did not vary by operator delay $[F(1,45)<1]$ or operand delay $\left[F(1,45)=2.89, M S_{\mathrm{e}}=0.049, p=.1\right.$; partial $\left.\eta^{2}=.06\right]$ in an operator delay $\times$ operand delay $\times$ block mixedfactorial ANOVA. A small, nonmonotonic effect of block was found $\left[F(8,360)=2.00, M S_{\mathrm{e}}=0.002, p<\right.$ .05 ; partial $\left.\eta^{2}=.04\right]$. The quadratic trend was significant $\left[F(1,45)=5.338, M S_{\mathrm{e}}=0.006, p<.05\right.$; partial $\left.\eta^{2}=.11\right]$, indicating that the participants demonstrated a slight decrease in accuracy, followed by a slight increase.

Another factor that could have affected the selected request and display orders is the side of the display on which information was requested and viewed. It is possible that the participants could have chosen to make requests that would have allowed them to read the information displays from left to right-for example, seeing the operands first when they were displayed to the left of the operators. Side-of-screen (left or right) one-way ANOVAs were run separately for request and display orders. No effect of side of screen was found for request order $[F(1,47)<1]$, although the participants with the operator on the left $(M=75 \%)$ were slightly more likely to request the operator first than were the participants with the operator on the right $(M=67 \%)$. No effect of side of screen was found for display order $(p>.2)$, although the participants for whom the operator was on the left $(M=71 \%)$ were slightly more likely to see the operator first than were the participants for whom the operator was on the right $(M=60 \%)$. Although the participants could have requested information so that they could read from left to right, this was not generally true. Instead, they typically requested and saw the operator first, regardless of the side of the screen on which it was displayed.

Individuals do seem to be sensitive to optimal sequencing when they must explicitly request information, but at least in this experiment, the participants tended not to achieve the optimal sequencing when environmental constraints made it difficult to do so. In the two short operator delay conditions, the participants almost always chose to see the operator first. Furthermore, the individuals in these groups became more likely to see the operator first with practice, suggesting that they were able to learn the optimal ordering of information. Because the delay between the operator request and the operator display was so short, it was relatively easy for the participants in these conditions to learn to optimally order the presentation of information.
Optimal sequencing seemed to be more difficult in the long operator delay/long operand delay condition. These participants saw the operator first less often than did the participants in the short operator delay/short operand delay and the short operator delay/long operand delay conditions. It is worth noting, however, that half (6 of 12) of the participants in this group made requests essentially simultaneously, never settling on a preferred request order. In these cases, the participants may have made requests simultaneously in an effort to see the most information as soon as possible, given the extended delay between their requests and the information displays. Of the participants in this condition who did establish a consistent request order, 4 of the 6 chose to request and see the operator before the operands.

The long operator delay/short operand delay condition resulted in the least frequent optimal ordering. The participants in this condition rarely saw the operator first. This was the condition in which it was most difficult to see the operator before the operands. For these participants, seeing the operator first would have meant waiting 1,500 msec before they saw any information at all. Sohn and Carlson (1998) found a benefit of approximately $200 \mathrm{msec}$ or less for participants who saw the operator first, as compared with those who saw the operands first. For the participants in the long operator delay/short operand delay condition, this benefit would have been more or less canceled by the additional time spent waiting for the operands. Many of these participants requested the operator first, then requested and saw the operands while they were waiting for the operator to appear (see Table 1). If they had waited for the operator to appear first, they then would have had to wait at least another $200 \mathrm{msec}$ before the presentation of the operands. Although seeing the operands before the operator may have been suboptimal, the participants in the long operator delay/long operand delay condition and the long operator delay/short operand delay condition may simply have adopted a strategy of seeing as much information as they could as soon as possible.

\section{EXPERIMENT 2}

In Experiment 1, timing constraints affected the participants' information acquisition strategies. Another factor that might be expected to influence the display order selected by participants is the feedback they receive. Previous work in the motor domain has shown that manipulating the type or frequency of feedback that participants receive can influence how well they are able to learn the required task (e.g., Wulf \& Schmidt, 1989). Accuracy in the present task was already quite high, so it was unlikely that altering feedback would affect accuracy. It would be reasonable to expect, however, that changing the solution time feedback might influence the strategy that the participants would choose, perhaps by encouraging the participants to optimize a certain portion of the total task. 
In previous research on the operator-first benefit (Carlson \& Sohn, 2000; Sohn \& Carlson, 1998), the time to complete each problem was measured from the time at which participants had all of the necessary information. In Experiment 1, however, the participants received feedback on the total time taken to complete each problem, which may have encouraged some participants to adopt less than optimal strategies. For example, half of the participants in the long operator delay/long operand delay condition requested both the operator and the operands at the same time, presumably to try to see the information as soon as possible. This strategy, however, may have led them to take more time than necessary after the presentation of that information to actually solve the problem. Solution time for this group of participants did decrease less with practice than did the time taken by the other groups. A block $\times$ operator $\times$ operand ANOVA on solution time revealed a significant three-way interaction $\left[F(8,360)=3.89, M S_{\mathrm{e}}=17,968, p<.001\right.$; partial $\left.\eta^{2}=.08\right]$. Follow-up analyses showed significant decreases in solution time with practice for all the groups except that in the long operator delay/long operand delay condition. Sohn and Carlson (1998) found that participants solved problems more slowly when operators and operands appeared simultaneously than when either was available in advance.

Experiment 2 was designed to examine the possibility that feedback that was more targeted would result in a more optimal strategy development. In this experiment, the participants received feedback on the basis of the time taken to solve each problem after they had seen both the operator and the operands. This measure reflects execution time primarily, plus the time needed to encode the last problem element to appear. We expected this change to increase the likelihood that optimal strategies would be developed, including seeing the operator before the operands and avoiding simultaneous requests for operator and operand information.

\section{Method}

Forty-eight participants were recruited from undergraduate psychology courses. One participant's data were lost due to computer error, resulting in 11 participants in the short operator delay/long operand delay condition and 12 participants in all the other conditions.

The method was identical to that in Experiment 1, except for the feedback given to the participants. As in Experiment 1, the participants received accuracy feedback for all trials and RT feedback for correct trials. In this experiment, the RT feedback was based on the time between the last piece of information presented and the time the answer was entered, and it was presented to the participants in terms of seconds.

\section{Results and Discussion}

In this experiment, the participants arranged their information requests so that they saw the operator first on a large majority of trials $(M=80 \%)$. They also became more likely to see the operator first with practice $[F(8,344)=$ 4.61, $M S_{\mathrm{e}}=0.09, p<.001$; partial $\left.\eta^{2}=.10\right]$, as was shown in an operator delay $\times$ operand delay $\times$ block mixed-factorial ANOVA. The proportion of operatorfirst displays increased more rapidly for the participants with a short operator delay than for the participants with a long operator delay $\left[F(8,344)=1.98, M S_{\mathrm{e}}=0.04, p<\right.$ .05 ; partial $\left.\eta^{2}=.04\right]$. The participants with short operand delays were less likely $(M=68 \%)$ to see the operator first than were those with long operand delays $\left[M=91 \% ; F(1,43)=5.79, M S_{\mathrm{e}}=5.56, p<.05\right.$; partial $\left.\eta^{2}=.12\right]$. No other display order effects were significant (all $p s>.1$ ). The proportions of trials in which the operators were displayed and requested first are shown in Table 1.

To further examine the effect of feedback on display order, we conducted an experiment $x$ operator delay $\times$ operand delay $\times$ block mixed-factorial ANOVA comparing Experiments 1 and 2. The participants in Experiment 2 were more likely to see the operator first than were the participants in Experiment $1[F(1,88)=6.43$, $M S_{\mathrm{e}}=4.19, p<.05$; partial $\left.\eta^{2}=.07\right]$. This was qualified by an operator delay $\times$ experiment interaction $\left[F(1,88)=14.75, M S_{\mathrm{e}}=9.62, p<.001\right.$; partial $\eta^{2}=$ $.14]$. Analyses of the simple main effects revealed no significant difference between experiments for the short operator delay conditions. When the operator delay was long, however, the participants in Experiment 2 were much more likely to see the operator first. The probability of seeing the operator first also increased more with practice in Experiment 2 than in Experiment $1[F(8,704)=$ $3.53, M S_{\mathrm{e}}=0.09, p=.001$; partial $\left.\eta^{2}=.04\right]$.

As in Experiment 1, request and display orders were necessarily the same for the participants in the equaldelay conditions. Request and display orders could differ, however, for the participants in the two mixed-delay conditions. Again, the interesting case is the short operator delay/long operand delay condition, in which the participants were expected to request the operands first but then to request and see the operator while waiting for the operands to appear. The participants in this condition requested the operator first on $53 \%$ of the trials, although they actually saw the operator first on $97 \%$ of the trials. Thus, on $44 \%$ of the trials, the participants requested the operands first but then requested and saw the operator while waiting for the operands to be displayed.

All the participants adopted consistent display orders. By the last two blocks of problems, all the participants had seen either the operator or the operands first on at least $85 \%$ of the trials. Thirty-nine consistently saw the operator first; 8 saw the operands first $\left[\chi^{2}(1, N=47)=\right.$ $20.45, p<.001]$.

To achieve these consistent display orders, most of these participants adopted a consistent request order. All but 4 participants requested either the operator or the operands first on more than $85 \%$ of the trials during the last two blocks. Thirty-four participants requested the operator first, whereas 9 consistently requested the operands first $\left[\chi^{2}(1, N=43)=14.54, p<.001\right]$. In contrast to Experiment 1, the participants in this experiment did not make simultaneous requests for information. 
Accuracy does not explain any differences in the ordering of information among conditions. Accuracy was high both before and after the introduction of the experimental manipulation. For the first block of trials, accuracy was high $(M=95 \%)$, and it did not vary as a function of operator delay $\left[F(1,43)=2.6, M S_{\mathrm{e}}=0.004\right.$, $p>.1$; partial $\left.\eta^{2}=.06\right]$ or operand delay $[F(1,43)<1]$, as was shown by an operator delay $X$ operand delay between-subjects ANOVA. The operator $\times$ operand delay interaction did not near significance $[F(1,43)<1]$. After the introduction of the experimental manipulation, accuracy remained high $(M=94 \%)$, and it did not vary by operator delay $\left[F(1,43)=1.28, M S_{\mathrm{e}}=0.011, p>\right.$ .25 ; partial $\left.\eta^{2}=.03\right]$, operand delay $[F(1,43)<1]$, or block $[F(8,344)<1]$.

The side of the display on which information was requested and displayed did have some effect on the selected request and display orders, as was shown by separate one-way side-of-screen ANOVAs on request order and display order. The participants who saw the operator on the left $(M=83 \%)$ were marginally more likely to request the operator first than were the participants who saw the operator on the right $[M=64 \% ; F(1,45)=3.38$, $M S_{\mathrm{e}}=0.44, p<.1$; partial $\left.\eta^{2}=.07\right]$. The participants for whom the operator was displayed on the left were also more likely to see the operator first $(M=92 \%)$ than were the participants who saw the operator on the right $\left[M=68 \% ; F(1,45)=6.06, M S_{\mathrm{e}}=0.66, p<.05\right.$; partial $\left.\eta^{2}=.12\right]$. This suggests that seeing the operator to the left of the operands makes participants more likely to make requests so that they see that piece of information first, presumably because of their experience with reading from left to right. However, the majority of the participants who saw operators to the right of the operands chose to see the operator first, even though that meant reading from right to left.

As in Experiment 1, the participants generally chose to see the operator before the operands, consistent with the optimal ordering. The feedback change did have an effect. The participants in this experiment were less likely to see both pieces of information at the same time, and they were more likely to see the operator first. Furthermore, they showed more evidence of skill acquisition, tending toward more optimal strategies with practice. The difference between experiments was perhaps most pronounced for the participants in the long operator delay/short operand delay condition. Because the feedback no longer contained any extra waiting time before the presentation of information, these participants were more likely to see the operator first, as compared with the same delay group in Experiment 1.

\section{EXPERIMENT 3}

Experiment 3 was designed to extend the findings of these experiments to a multiple-step task. Each step consisted of a single mathematical operation. During the first step, the participant saw one operator and two operands. The result of that step served as an operand for the subsequent step. For the remainder of the steps, the participants saw an operator and one new operand, using the result from the previous step as the second operand. After the eighth and final step of the problem, the participants entered their final answer.

In this experiment, the participants received feedback only on the total time to complete all eight steps of each problem. We expected, however, that the additional demands of this task - the need to hold intermediate results for subsequent use-would also encourage information acquisition strategies consistent with optimal sequencing. Viewing a new operand before seeing the operator places an additional burden on working memory, requiring that the new operand be stored together with the previous result until the operator is available. We therefore expected to find once again that the participants would generally choose to view operators before operands, even when timing constraints made this ordering relatively difficult to achieve and latency feedback did not focus primarily on execution time.

\section{Method}

Fifty-two participants were recruited from the psychology participant pool. The participants were randomly assigned to the same four delay conditions as those in Experiments 1 and 2.

The display that the participants saw at the beginning of each problem is shown in Figure 1. This problem frame remained visible throughout the problem. The operands appeared within the parentheses. The operators appeared to either the immediate left or the immediate right of the parentheses, and this was counterbalanced across participants, as in Experiments 1 and 2 .

The first block of problems was similar to the first block in Experiments 1 and 2. The participants used the space bar to make information requests, and the operator and the operand for each step appeared at the same time. Again, this introduced the participants to the task and served as a check on random assignment. For the remaining blocks of problems, the participants requested the operators and operands at each step of the problem, as in the previous experiments.



Figure 1. Beginning problem display for Experiment 3. 
The operators were the same as those in Experiments 1 and 2, and each operator appeared twice in each trial. The order of the operators was random. Operands ranging from one to nine were randomly selected, with the constraint that the answer to each step of the problem had to be a single-digit number. If the computer generated a sequence of operators and operands in which an answer to any step of the problem was greater than nine, a new problem was generated until this condition was met.

The participants completed a total of 10 blocks of 10 trials each, including the practice trial. They received accuracy feedback at the end of each trial and RT feedback at the end of correct trials. RT feedback was the total time, in seconds, to complete the problem (from the first request for information to the time at which the final answer was entered).

\section{Results and Discussion}

The participants saw the operator before the operand on the majority of steps $(M=71 \%)$. An operator delay $\times$ operand delay $\times$ block mixed-factorial ANOVA was conducted on display order. The participants in the short operator delay conditions saw the operator first more often $(M=83 \%)$ than did the participants in the long operator delay conditions $[M=59 \% ; F(1,48)=4.86$, $M S_{\mathrm{e}}=6.39, p<.05$; partial $\left.\eta^{2}=.09\right]$. In the long operator delay/short operand delay condition, the participants saw the operator first $60 \%$ of the time, a much larger percentage of steps than in the comparable group in Experiment 1. With practice, the participants became more likely to see the operator first $[F(8,384)=2.62$, $M S_{\mathrm{e}}=0.04, p<.01 ;$ partial $\left.\eta^{2}=.05\right]$, and this effect of block did not interact with either the operator or the operand delay conditions $(p s<1)$. In the first block of trials following the experimental manipulation, the participants saw the operator first on $64 \%$ of the steps. By the last block of problems, the participants saw the operator first on $74 \%$ of the steps. No other display order effects were significant (all $p \mathrm{~s}>.1$ ). The proportions of steps in which the operators were displayed and requested first are shown in Table 1.

As in Experiments 1 and 2, display and request orders were necessarily the same only for the two equal operator delay/operand delay conditions. Again, the participants in the short operator delay/long operand delay condition sometimes requested the operand first but then requested and saw the operator before the operand. These participants requested the operator first on an average of $66 \%$ of the steps, although they actually saw the operator first on $82 \%$ of the steps. This means that on $16 \%$ of the steps, the participants saw the operator while waiting for the operand display.

Most of the participants established a consistent display order. A total of 46 (of 52) participants saw either the operator or the operand first on more than $85 \%$ of the steps by the last two blocks of trials. Thirty-five of these participants consistently saw the operator first; only 11 consistently saw the operand first. This was found to differ significantly from chance $\left[\chi^{2}(1, N=46)=12.52\right.$, $p<.001]$.

To accomplish these consistent display orders, most of the participants established consistent request orders.
All but 8 participants consistently requested either the operator or the operand first on at least $85 \%$ of the steps. Of the 44 participants who consistently requested either the operator or the operand first, 33 consistently requested the operator first, and 11 consistently requested the operand first $\left[\chi^{2}(1, N=44)=11.00, p=.001\right]$.

Accuracy does not explain any group differences among request or display orders. The participants were reasonably accurate both before and after the introduction of the experimental manipulation. In the first block of trials, accuracy did not differ as a function of operator delay or operand delay $[M=84 \% ; F(1,48)<1]$. After the introduction of the experimental manipulation, accuracy remained at approximately $84 \%$ of the trials, and it did not vary by operator or operand delay $[F(1,48)<1]$.

The side of the screen on which the operators appeared affected request order $\left[F(1,50)=9.41, M S_{\mathrm{e}}=\right.$ $1.2, p<.01$; partial $\left.\eta^{2}=.16\right]$. The participants who saw the operator on the left side of the screen were more likely to request the operator first $(M=86 \%)$ than were the participants who saw the operator on the $\operatorname{right}(M=$ $57 \%$ ). A marginally significant effect was found for display order $\left[F(1,50)=3.69, M S_{\mathrm{e}}=0.53, p=.06\right.$; partial $\left.\eta^{2}=.07\right]$. The participants who saw the operator on the left were marginally more likely to see the operator first $(M=81 \%)$ than were the participants who saw the operator on the right $(M=61 \%)$. This numerically large but statistically marginal difference can be explained by a high level of interparticipant variability. Overall, the participants tended to request and see the operator first regardless of whether the information was displayed on the right or the left of the screen. The participants did not simply choose to see the information so that they could read from left to right.

The results of this experiment supported the hypothesis that the participants would organize procedures in an optimal way. The participants in all four conditions elected to view the operator before the operand most of the time. A greater than chance number of participants consistently chose to see the operator before the operand. Even though feedback was not provided on a step-bystep basis, most of the participants still adopted the optimal display order, avoiding simultaneous information displays.

\section{GENERAL DISCUSSION}

The results from these three experiments demonstrate that people are able to structure and sometimes adjust their strategies for picking up information on the basis of their own fine-grained structure of cognitive processes for arithmetic. In all three experiments, the participants timed and sequenced their requests so that the operator was displayed before the operands the majority of the time. Furthermore, a greater number of participants than would be expected by chance consistently saw the operator first by the end of practice. This display order matches the order described by the procedural frame hy- 
pothesis (Carlson \& Sohn, 2000; Sohn \& Carlson, 1998). In some cases, this optimal ordering became more likely with practice. The likelihood of achieving this optimal ordering, however, depended on the difficulty of displaying the information in the optimal order, the type of feedback received, and the complexity of the task.

The participants were able to organize their information requests so that the displayed information was compatible with the underlying cognitive structure of the task, as described by the procedural frame hypothesis. On the basis of the procedural frame hypothesis and previous research (Carlson \& Sohn, 2000; Sohn \& Carlson, 1998), this ordering of information was likely to allow participants to solve problems up to $200 \mathrm{msec}$ or so faster than they would have had they seen the operands first. The participants' selection of their own display orders in the present experiments precluded the inferential examination of possible benefits here. Inspection of the data does suggest small advantages when the participants saw the operator first, however. In Experiments 1 and 2, trials in which the operator was seen first were solved approximately 15 and $250 \mathrm{msec}$ faster, respectively, than trials in which the operands were seen first. In Experiment 3, the participants who typically chose to see the operator first requested the first piece of information for the subsequent step about $140 \mathrm{msec}$ before the participants who consistently saw the operand first did. This finding that the participants chose to adopt the strategy of seeing the operator before the operands is similar to Gray and Boehm-Davis's (2000) finding that participants do make changes in strategy that result in very small benefits in performance time. In their work, this benefit was on the order of 100-200 msec.

The results of these experiments suggest that individuals adapt their information acquisition strategies to fine-grained cognitive structure when the advantage of doing so is salient. Some previous literature has suggested that people adjust strategies to minimize demands on working memory even when those demands fall far short of working memory capacity. For example, Ballard, Hayhoe, and Pelz (1995) examined the use of working memory in a task that involved copying colored blocks. They found that participants did not work at the limits of short-term memory capacity, preferring instead to pick up information just before it was needed for the task. This is similar to the results we found in Experiment 3, in which the participants ordered the display of information in a way that would minimize demands on working memory, giving them the information as it was needed.

Although overall, the participants did choose to see the information in the optimal order, there was a sizable minority of participants who consistently saw the operand first. The costs of not adopting the optimal strategy are somewhat slower RTs and somewhat increased cognitive effort. These costs were enough for the majority of the participants to develop the optimal strategy, but this group may simply not have chosen to invest the effort into discovering the optimal strategies. Most of the participants did settle on one strategy very early, and they did not adjust this strategy with practice.

The consideration of information acquisition strategies in the present experiments should be distinguished from that in previous work on the role of execution strategies in arithmetic. In contrast to the present study, previous work on strategies in arithmetic has focused on how individuals generate solutions, given that operator and operand information is available. For example, Siegler and Shrager (1984) found that children who are learning to add may select from several different strategies, such as counting and retrieval. Although children may sometimes confuse operations as they acquire skill in arithmetic (e.g., Miller \& Paredes, 1990), research on arithmetic execution strategies generally has assumed that the goal of performing a particular operation is encoded and available before operands are considered (e.g., Widaman, Geary, Cormier, \& Little, 1989). Alternatively, some models (e.g., Reder \& Schunn, 1996; Siegler \& Shrager, 1984) propose that particular problems (e.g., $3+5$ ) are associated in memory with their answers, suggesting that operators and operands serve similar roles as retrieval cues. The present work does not address the issue of how execution procedures are selected, but our account is compatible with any view that assumes that arithmetic operators are considered prior to operands.

Unlike the strategy selection we have described in the present work, execution strategies do not seem to be influenced by increased working memory demands. Hecht (2002) found that the proportion of trials in which participants chose a retrieval strategy remained consistent regardless of different working demands. In contrast, we found that participants were more likely to use an operatorfirst strategy when we increased the working memory requirement in Experiment 3.

One question that is common to work on execution strategies and the present work on information acquisition strategies concerns the extent to which strategy development and selection is implicit or deliberate. For example, Reder and Ritter (1992) have argued that the choice of execution strategies for solving arithmetic problems is implicit. Reder and Schunn (1996) later argued that selection of execution strategies for a variety of cognitive tasks is generally implicit. An important but unanswered question is how the participants selected their information acquisition strategies in the present experiments. The participants may have used an explicit representation of the strategy space, deliberately figuring out the best strategy to use. Alternatively, they could have implicitly explored the strategy space over trials and selected the strategy that gave the best results. The need for deliberate action to display information and the increase in the selection of optimal display sequences under difficult conditions when, in Experiment 2, feedback emphasized execution time suggest a deliberate compent to strategy selection. However, the present data do not address this question directly. 
The present work can also be compared with research on the role of information order in the following of written instructions. Dixon (1987a, 1987b; Dixon, Faries, \& Gabrys, 1988) examined information order in terms of whether component or organizational information came first, whether implicit or explicit information came first, and whether the antecedent or the consequence of the action came first. Dixon presented a model suggesting that people interpret written directions fastest if they see organizational information first, then fill in lower level details. This proposal resembles the procedural frame hypothesis, which suggests that goals must be considered first and the arguments to that goal later filled in. In both accounts, the information considered first provides a schematic frame to which additional information is assimilated. In Dixon's work, however, higher level organizational knowledge and lower level component knowledge were really two different levels of description of the same information (cf. Vallacher \& Wegner, 1987). For example, people followed directions more quickly when reading, "Draw a wagon by drawing a rectangle with two circles under it" than when reading, "Draw a rectangle with two circles under it to draw a wagon." In the first case, the organizational knowledge preceded the component knowledge, whereas the reverse was true in the second case. In both cases, however, the organizational and the component information specify the same action, at different levels. One could presumably complete the task with reasonable accuracy when given only the organizational knowledge ("draw a wagon") or the component knowledge ("draw a rectangle with two circles under it"). In contrast, operator and operand information are both necessary to complete the arithmetic tasks studied here.

The present results extend previous work demonstrating that individuals adjust their cognitive strategies to achieve small latency benefits (Gray \& Boehm-Davis, 2000). They also support the contention that individuals use deictic representations to realize "just-in-time" representations that minimize working memory demands (Ballard et al., 1997). Even at the fine-grained level of individual steps of cognitive activity, individuals can overcome environmental constraints to adopt information acquisition strategies that reflect the structure of cognitive processes. More generally, these results support the suggestion that temporal coordination with the environment is a critical feature of cognitive, as well as perceptual-motor, skill (Carlson, 1997; Neisser, 1983; Rosenbaum, Carlson, \& Gilmore, 2001) and that the study of cognitive skill should include examination of information acquisition strategies.

\section{REFERENCES}

Ballard, D. H., Hayhoe, M. M., \& Pelz, J. B. (1995). Memory representations in natural tasks. Journal of Cognitive Neuroscience, $\mathbf{7}$, 66-80.

Ballard, D. H., Hay hoe, M. M., Pook, P. K., \& Rao, R. P. N. (1997).
Deictic codes for the embodiment of cognition. Behavioral \& Brain Sciences, 20, 723-767.

BovaIR, S., \& KIERAS, D. E. (1991). Toward a model of acquiring procedures from text. In R. Barr, M. L. Kamil, P. Mosenthal, \& P. D. Pearson (Eds.), Handbook of reading research II (pp. 206-229). White Plains, NY: Longman.

CARlson, R. A. (1997). Experienced cognition. Mahwah, NJ: Erlbaum. CArlson, R. A., \& Sohn, M.-H. (2000). Cognitive control of multiplestep routines: Information processing and conscious intentions. In S. Monsell \& J. Driver (Eds.), Control of cognitive processes: Attention and performance XVIII (pp. 443-464). Cambridge, MA: MIT Press.

CARY, M., \& CARlson, R. A. (1999). External support and the development of problem solving routines. Journal of Experimental Psychology: Learning, Memory, \& Cognition, 25, 1053-1070.

Clark, J. M., \& CAmpBell, J. I. D. (1991). Integrated versus modular theories of number skills and acalculia. Brain \& Cognition, 17, $204-$ 239.

Dehaene, S. (1992). Varieties of numerical abilities. Cognition, 44, 1-42.

Dixon, P. (1987a). The processing of organizational and component step information in written directions. Journal of Memory \& Language, 26, 24-35.

Dixon, P. (1987b). The structure of mental plans for following directions. Journal of Experimental Psychology: Learning, Memory, \& Cognition, 13, 18-26.

Dixon, P., FARIES, J., \& GABrys, G. (1988). The role of explicit action statements in understanding and using written directions. Journal of Memory \& Language, 27, 649-667.

Gray, W. D., \& Boehm-Davis, D. A. (2000). Milliseconds matter: An introduction to microstrategies and to their use in describing and predicting interactive behavior. Journal of Experimental Psychology: Applied, 6, 322-335.

HeCHT, S. A. (2002). Counting on working memory in simple arithmetic when counting is used for problem solving. Memory \& Cognition, 30, 447-455.

Just, M. A., \& CARpenter, P. A. (1980). A theory of reading: From eye-fixations to comprehension. Psychological Review, 87, 329-354.

Miller, K. F., \& Paredes, D. R. (1990). Starting to add worse: Effects of learning to multiply on children's addition. Cognition, 37, 213242.

NeISSER, U. (1983). Toward a skillful psychology. In D. R. Rogers \& J. A. Sloboda (Eds.), Acquisition of symbolic skills (pp. 1-17). New York: Plenum.

PAshler, H., \& Johnston, J. C. (1989). Chronometric evidence for central postponement in temporally overlapping tasks. Quarterly Journal of Experimental Psychology, 41, 19-45.

PAYNE, J. W. (1976). Task complexity and contingent processing in decision making: An information search and protocol analysis. Organizational Behavior \& Human Decision Processes, 16, 366-387.

Reder, L. M., \& RitTer, F. E. (1992). What determines initial feeling of knowing? Familiarity with question terms, not with the answer. Journal of Experimental Psychology: Learning, Memory, \& Cognition, 18, 435-451.

Reder, L. M., \& Schunn, C. (1996). Metacognition does not imply awareness: Strategy choice is governed by implicit learning and memory. In L. M. Reder (Ed.), Implicit memory and metacognition (pp. 45-77). Mahwah, NJ: Erlbaum.

Rogers, R. D., \& Monsell, S. (1995). Costs of a predictable switch between simple cognitive tasks. Journal of Experimental Psychology: General, 124, 207-231.

Rosenbaum, D. A., Carlson, R. A., \& Gilmore, R. O. (2001). Acquisition of intellectual and perceptual-motor skills. Annual Review of Psychology, 52, 453-470.

SCHNeIdER, W. (1988). Micro Experimental Laboratory: An integrated system for IBM PC compatibles. Behavior Research Methods, Instruments, \& Computers, 20, 206-217.

Shin, J. C., \& Rosenbaum, D. A. (2002). Reaching while calculating: Scheduling of cognitive and perceptual-motor processes. Journal of Experimental Psychology: General, 131, 206-219.

Siegler, R. S., AdOLPH, K. E., \& Lemaire, P. (1996). Strategy choices 
across the life span. In L. M. Reder (Ed.), Implicit memory and metacognition (pp. 79-121). Mahwah, NJ: Erlbaum.

Siegler, R. S., \& Shrager, J. (1984). Strategy choices in addition and subtraction: How do children know what to do? In C. Sophian (Ed.), Origins of cognitive skill (pp. 229-293). Hillsdale, NJ: Erlbaum.

SoHN, M.-H., \& CARLSON, R. A. (1998). Procedural frameworks for simple arithmetic skills. Journal of Experimental Psychology: Learning, Memory, \& Cognition, 24, 1052-1067.

Sohn, M.-H., \& CARLSON, R. A. (2000). Effects of repetition and foreknowledge in task-set reconfiguration. Journal of Experimental Psychology: Learning, Memory, \& Cognition, 26, 1445-1460.

Vallacher, R. R., \& Wegner, D. M. (1987). What do people think they're doing? Action identification and human behavior. Psychological Review, 94, 3-15.

Widaman, K. F., Geary, D. C., Cormier, P., \& Little, T. D. (1989). A componential model for mental addition. Journal of Experimental Psychology: Learning, Memory, \& Cognition, 15, 898-919.

WULF, G., \& SCHMIDT, R. A. (1989). The learning of generalized motor programs: Reducing the relative frequency of knowledge of results enhances memory. Journal of Experimental Psychology: Learning, Memory, \& Cognition, 15, 748-757.

(Manuscript received June 11, 2002;

revision accepted for publication August 3, 2003.) 